Bull. Austral. Math. Soc.

$16 \mathrm{~A} 21,16 \mathrm{~A} 12$

Vor. 49 (1994) [55-57]

\title{
A NOTE ON PRIME ESSENTIAL RINGS
}

Hossein ZaND

It is proved that the upper radical determined by the class of prime essential rings is an $N$-radical.

Several examples are known of hereditary supernilpotent radicals which are not special. Recently Gardner and Stewart [2] showed how one can construct many such examples (including most of those already known) using prime essential rings, which were introduced for other reasons by Rowen in [5]. In particular Gardner and Stewart showed that the upper radical determined by the class of these rings is not special. The aim of this note is to prove that this upper radical is also an $N$-radical. Note that the first example of an $N$-radical which is not special was found by Jaegermann and Sands $[3]$.

All rings in this paper are associative, not necessarily with 1 . To denote that $I$ is an ideal (prime ideal, left ideal) of a ring $R$ we write $I \triangleleft R\left(I \triangleleft^{\prime} R, I<R\right)$. An ideal $I$ of a ring $R$ is called essential (in $R$ ) if for every $0 \neq J \triangleleft R, I \cap J \neq 0$.

A ring $R$ is called prime essential [5] if it is semiprime and all its prime ideals are essential.

Recall that a radical is an $\boldsymbol{N}$-radical [6] if it is left strong (that is, semisimple rings contain no non-zero radical left ideals), left hereditary (that is, left ideals of radical rings are radical) and contains the prime radical $\beta$. Throughout the paper instead of "radical class" we write briefly "radical".

All undefined terms and facts used can be found in [7].

We start with some lemmas.

LEMMA 1. [2]

(i) Every ideal of a prime essential ring is a prime essential ring.

(ii) If $I$ is an essential ideal of $R$ and $I$ is a prime esssential ring then $R$ is a prime essential ring.

Received 1st February, 1993

The author wishes to thank Professor Puczylowski for his valuable help.

Copyright Clearance Centre, Inc. Serial-fee code: 0004-9729/94 \$A2.00+0.00. 
Lemma 2. [6, Corollary p. 338]. If $L<R$ and $R$ is a semiprime ring then $\beta(L)=\{l \in L \mid L l=0\}$.

Lemma 3. Let $I \triangleleft L<R$ and let $L / I$ be a semiprime ring. If $M$ is the largest ideal of $R$ such that $M L \subseteq I$ then the ring $R / M$ is semiprime and $L I \subseteq M$.

Proof: If $M \varsubsetneqq J \triangleleft R$ then by the choice of $M, J L \nsubseteq I$. Obviously $J L$ is an ideal of $L$, so since $L / I$ is semiprime, $(J L)^{2} \nsubseteq I$. But $(J L)^{2} \subseteq J^{2}$, so $J^{2} \neq 0$. This shows that the ring $R / M$ is semiprime.

Notice that $L I R$ is an ideal of $R$ and $L I R L \subseteq I$. Consequently $L I R \subseteq M$. Since $R / M$ is semiprime, $L I \subseteq M$.

The following lemma can be easily deduced from results in [6]. We include its direct proof for completeness.

Lemma 4. Let $L<R$.

(i) If $I \triangleleft L$ then $\bar{I}=\{r \in R \mid R r L \subseteq I\} \triangleleft R$ and if $I \triangleleft^{\prime} L$ then $\bar{I} \triangleleft^{\prime} R$.

(ii) If $I \triangleleft R$ then $I^{*}=\{l \in L \mid L l R \subseteq I\} \triangleleft L$ and if $I \triangleleft^{\prime} R$ then $I^{*} \triangleleft^{\prime} L$.

Proof: (i) The first part of the statement is clear. Suppose now that $J, K$ are ideals of $R$ and $J K \subseteq \bar{I}$. Then $R J K L \subseteq I$. Obviously $J L R K \subseteq J K$, so $R J L R K L \subseteq$ $I$. However $R J L$ and $R K L$ are ideals of $L$. Hence, since $I$ is a prime ideal of $L$, $R J L \subseteq I$ or $R K L \subseteq I$. Consequently $J \subseteq \bar{I}$ or $K \subseteq \bar{I}$ and the result follows.

(ii) It is clear that $I^{*}$ is an ideal of $L$. Suppose now that $I \triangleleft^{\prime} R$ and $J, K$ are ideals of $L$. If $J K \subseteq I^{*}$ then $L J K R \subseteq I$. Obviously $L J R L K R \subseteq L J K R \subseteq I$ and $L J R, L K R$ are ideals of $R$. Since $I$ is a prime ideal of $R, L J R \subseteq I$ or $L K R \subseteq I$. Consequently $J \subseteq I^{*}$ or $K \subseteq I^{*}$ and the result follows.

Recall that a class $T$ of rings is called regular if every non-zero ideal of each ring in $T$ can be homomorphically mapped onto a non-zero ring in $T$. The upper radical determined by $T$ is equal the class of all rings which cannot be homomorphically mapped onto non-zero rings in $T$.

Lemma 5. [1] Given a regular class $T$ of rings, the upper radical $U_{T}$ determined by $T$ is left strong if and only if every non-zero left ideal of each ring in $T$ can be homomorphically mapped onto a non-zero ring in $T$.

Now we are ready to prove

THEOREM 6. The upper radical $U_{\mathcal{E}}$ determined by the class $\mathcal{E}$ of prime essential rings is an $N$-radical.

Proof: Lemma 1 implies that the class $\mathcal{E}$ is regular. Thus to prove that $U_{\mathcal{E}}$ is left strong we can apply Lemma 5. We shall show that if $0 \neq L<R$ and $R$ is prime essential then $L / \beta(L)$ is prime essential. For, let $M$ be an ideal of $R$ maximal with respect to $M \cap\langle L\rangle=0$, where $\langle L\rangle=L+L R$. Observe that $\langle L\rangle+M / M$ is an essential ideal of 
$R / M$. Therefore by Lemma $1, R / M$ is prime essential and without loss of generality, we may assume that $M=0$. Now take ideals $P$ and $I$ of $L$ such that $\beta(L) \subseteq P \triangleleft^{\prime} L$ and $\beta(L) \subsetneq I \triangleleft L$. By Lemma 2, $L I \neq 0$. Hence, since $R$ is semiprime, $L I R \neq 0$. Observe that $L I R \subseteq \bar{I}$, so $\bar{I} \neq 0$. By Lemma 4, $\bar{P} \triangleleft^{\prime} R$. Thus $\bar{I} \cap \bar{P} \neq 0$. Since $\langle L\rangle$ is an essential ideal of $R$ and $R$ is semiprime $((I \cap \bar{P}) \cap\langle L\rangle)^{2} \neq 0$. This in particular implies that $L R(I \cap \bar{P}) L \neq 0$, which together with Lemma 2 gives $R(\bar{I} \cap \bar{P}) L \nsubseteq \beta(L)$. Hence since $R(\bar{I} \cap \bar{P}) L \subseteq I \cap P$, we get $I \cap P \nsubseteq \beta(L)$. This proves that $L / \beta(L)$ is prime essential.

Now we shall show that $U_{\mathcal{E}}$ is left hereditary. If not then there exist a ring $R \in U_{\mathcal{E}}$ and $I \unlhd L<R$ such that $0 \neq L / I \in \mathcal{E}$. Let $M$ be the largest ideal of $R$ satisfying $M L \subseteq I$. Notice that since $I$ is a semiprime ideal of $L, M \cap L \subseteq I$. Consequently by passing to the factor ring $R / M$ we can assume without loss of generality that $M=0$. By Lemma $3, R$ is a semiprime ring and $L I=0$. This obviously gives $\beta(L)=I$. Let $P \triangleleft^{\prime} R$ and $0 \neq J \triangleleft R$. By Lemma 4, $P^{*} \triangleleft^{\prime} L$ and $J^{*} \triangleleft L$. Moreover $J L \subseteq J^{*}$ and by the foregoing, $J L \nsubseteq I$. Hence, since $L / I$ is prime essential, $P^{*} \cap J^{*} \nsubseteq I$. Consequently by Lemma 2, $L\left(P^{*} \cap L^{*}\right) \neq 0$. Further, since $R$ is semiprime, $L\left(P^{*} \cap J^{*}\right) R \neq 0$. However $L\left(P^{*} \cap J^{*}\right) R \subseteq P \cap J$, so $P \cap J \neq 0$. This proves that $R$ is prime essential, a contradiction.

REMARK. From the proof it also follows that the class of prime essential rings is normal [4].

\section{REFERENCES}

[1] N. Divinsky, J. Krempa and A. Suliński, 'Strong radical properties of alternative and associative rings', J. Algebra 17 (1971), 369-381.

[2] B.J. Gardner and P.N. Stewart, 'Prime essential rings', Proc. Edinburgh Math. Soc. 34 (1991), 241-250.

[3] M. Jaegermann and A.D. Sands, 'On normal radicals, N-radicals and A-radicals', J. AL gebra 50 (1978), 337-349.

[4] E.R. Puczylowski, 'On normal classes of rings', Comm. Algebra 20 (1992), 2999-3013.

[5] L.H. Rowen, 'A subdirect decomposition of semiprime rings and its application to maximal quotient rings', Proc. Amer. Math. Soc. 46 (1974), 176-180.

[6] A.D. Sands, 'Radicals and Morita contexts', J. Algebra 24 (1973), 335-345.

[7] R. Wiegandt, Radical and semisimple classes of rings, Queen's Papers in Pure and Applied Mathematics No. 37 (Queen's University, Kingston, Ontario, 1974).

Faculty of Mathematics

The Open University

Walton Hall

Milton Keynes MK7 6AA

England
Institute of Mathematics and Physics

Technical University of Białystok

15-351 Białystok

Wiejska 45A

Poland 